\title{
Raciocínio clínico do estudante de Enfermagem: análise a partir de um simulador virtual
}

\author{
Michele Antunes - FEEVALE - micheleantunes@gmail.com \\ Marta Bez - FEEVALE - martabez@feevale.br \\ Gabriela Perry - UFRGS - gabrielaperry@hotmail.com \\ Marie Jane Soares Carvalho - UFRGS - mariejse@gmail.com
}

\begin{abstract}
Resumo: O raciocínio clínico caracteriza-se por ser composto por um conjunto de atitudes ou hábitos, sendo classificado como habilidades e disposições cognitivas. $\mathrm{O}$ objetivo geral desta pesquisa foi analisar o raciocínio clínico dos estudantes de enfermagem a partir da aplicação de um simulador virtual, assim como identificar a perspectiva dos estudantes quanto ao seu percurso cognitivo. Trata-se de um estudo de delineamento transversal, com abordagem quantitativa , realizado com 40 estudantes em uma universidade do Sul do Brasil. A análise permitiu identificar que eles acreditam possuírem maiores conhecimentos do que foram verificados pelo simulador, no que se refere aos elementos: Capacidade de análise $(p=0,039)$, Discernimento $(p=0,004)$ e Conhecimento sobre o paciente $(\mathrm{p}=0,041)$. $\mathrm{O}$ ensino e a aprendizagem destas habilidades favorecem a construção da competência do estudante.
\end{abstract}

Palavras-chave: Educação em enfermagem. Simulação por computador. Pensamento crítico.

\section{Clinical reasoning of the nursing student: analysis from a virtual simulator}

Abstract: Clinical reasoning is characterized by being a set of attitudes or habits, being classified as cognitive abilities and dispositions. The general objective was to analyze the clinical reasoning of nursing students from the application of a virtual simulator, as well as to identify the students' perspective regarding their cognitive path. This is a crosssectional study, with a quantitative approach, carried out with 40 students. The analysis made it possible to identify that they believe they have greater knowledge, than was verified by the simulator, with regard to the elements: Ability to analyze $(p=0.039)$, Discernment $(p=0.004)$ and Knowledge about the patient $(p=0.041)$. The teaching and learning of these skills favor the construction of the student's competence.

Keywords: Nursing education. Computer simulation. Critical thinking.

\section{Introdução}

Os enfermeiros lidam com respostas a problemas de saúde e processos da vida entre indivíduos, famílias, grupos e comunidades. Essas respostas, são a preocupação central dos cuidados de enfermagem e ocupam o círculo atribuído à profissão (Gallagherlepak, 2018).

A tomada de decisão clínica, diagnóstica ou terapêutica, consiste nos processos do pensamento que compõem o raciocínio clínico (Carvalho; Oliveira-kumakura; Morais, 2017). Este, consiste em uma habilidade primordial na prática de enfermagem, 
pois é por meio dele que os profissionais identificam, priorizam, estabelecem planos e avaliam dados (Fitzpatrick; Smith, 2013).

Conforme Carbogim et al. (2019), existe a necessidade que o enfermeiro realize a análise e a interpretação, na busca pela avaliação e detalhamento de uma história clínica com a observação e relação de dados do paciente. É fundamental para que ele possa compreender as prioridades de necessidades de saúde a partir de um agrupamento de principais sinais e sintomas.

A capacidade de identificar as conclusões lógicas e verdadeiras sobre situações ou experiências, possibilitam a identificação e definição dos elementos estruturais que, na sua avaliação, conduzirão à tomada de decisão clínica. Entretanto, o ensino das habilidades que compõe o raciocínio clínico, precisa fazer parte do processo de formação do estudante de Enfermagem, pois é necessário que as lacunas de conhecimento sejam identificadas, e que a partir disso, estratégias de intervenção possam ser aplicadas.

Assim, a utilização de metodologias ativas de ensino-aprendizagem, aliada à promoção de ambientes facilitadores em sala de aula e no contexto da prática clínica, contribui para que o estudante de enfermagem possa desenvolver competências (Mártires et al., 2019). Diante deste contexto, entende-se que é importante preparar estudantes em realidades simuladas, virtuais e com manequins, para quando tiverem demonstrado habilidade e segurança serem encaminhados para realizar o procedimento com humanos.

Esse método permite o autorreconhecimento de eventuais erros antes de realizar o cuidado com o paciente real. As modalidades híbridas de ensino, que integram as Tecnologias Educacionais Digitais (TED) e manequins em ambientes simulados, proporcionam métodos flexíveis que desenvolvem o pensamento crítico e priorizam o protagonismo do estudante (Silveira; Cogo, 2017). Diante destas tecnologias, destaca-se o uso da simulação virtual no ensino da Enfermagem.

No estudo de Forsberg e colaboradores (2019), os estudantes relataram que a simulação virtual desenvolveu uma melhor compreensão da doença e uma visão mais holística do paciente, assim como um melhor entendimento do curso dos acontecimentos. O caso clínico do paciente virtual convidou os alunos a pensarem sobre como os detalhes estão conectados. Os alunos relataram que a simulação virtual ensinou a importância de não se concentrar apenas na suspeita inicial e de buscar soluções alternativas.

No estudo de Dubovi (2019), a utilização do simulador virtual contribuiu para um aumento de $12,9 \%$ nos escores do raciocínio clínico, considerando que foram avaliados itens como a capacidade do estudante de buscar as pistas certas na investigação e a ação correta. Para Betts et al. (2020), no simulador virtual os estudantes podem interagir com o sistema, com os cenários, escolher uma avaliação ou intervenção, e a sua escolha é então realizada, sendo fornecida uma resposta apropriada. Isso permite que os alunos continuem com sua avaliação ou plano de tratamento ou reavaliam suas decisões e mude $\mathrm{m}$ de direção na maneira como prestam assistência. Os alunos podem escolher um banco de cenários criados pelo desenvolvedor da ferramenta, como pacientes com problemas respiratórios, e podem definir a duração do cenário.

Esse contexto da simulação em enfermagem, que atualmente é retratado em publicações, pode contribuir para aprimorar as dinâmicas ativas de ensino, bem como, estimular novos estudos que possam melhorar os processos de ensino-aprendizagem junto aos estudantes. Tendo em vista as vantagens que muitos autores atribuem ao simulador virtual, a indagação da autora remete-se a analisar os elementos constituintes do pensamento crítico e o percurso cognitivo de estudantes de enfermagem durante a realização do raciocínio clínico na resolução do caso clínico com o uso desta ferramenta.

A utilização desse tipo de simulação tem como vantagem o uso de um formalismo que permite a criação de simulações nas quais o professor não necessita conhecer 
informática para o desenvolvimento do material pedagógico. O foco está na criação dos casos clínicos, e não no conhecimento de programação ou tarefas complexas de informática (BEZ et al., 2018).

Atualmente, identifica-se uma lacuna de conhecimento na literatura em enfermagem sobre os instrumentos validados e adaptados transculturalmente para $o$ idioma português e que mensurem quantitativamente o raciocínio clínico. Tendo em vista essa ausência de estudos, optou-se por realizar esta fase da pesquisa de forma a identificar as categorias de análise de cada uma das habilidades que o estruturam, relacionando-as com o percurso do estudante durante a utilização das ferramentas.

Desse modo, o objetivo geral deste estudo foi analisar o raciocínio clínico dos estudantes de enfermagem a partir da aplicação de um simulador virtual, assim como, identificar a perspectiva dos estudantes quanto ao seu percurso cognitivo a partir da aplicação do simulador virtual. Para tanto, a sessão dois apresenta os materiais e métodos empregados, na sessão três os resultados são demonstrados e discutidos, seguido das conclusões.

\section{Materiais e Métodos}

Trata-se de um estudo de delineamento transversal, com abordagem quantitativa, realizado em uma universidade do sul do país, com 40 estudantes de enfermagem matriculados na $6^{\mathrm{a}}, 7^{\mathrm{a}}$ e $8^{\mathrm{a}}$ etapa do curso, em 2020/1.

Os critérios de inclusão foram: a) ter no mínimo 18 anos completos; b) estar matriculado a partir do $6^{\circ}$ semestre do curso de bacharelado em enfermagem; c) ter concluído com aprovação a disciplina "Enfermagem na Saúde do Adulto II"; d) ter concluído com aprovação a disciplina "Estágio Curricular I"; e) estar matriculado regularmente nas disciplinas "Prática Supervisionada de Enfermagem na Assistência ao Paciente Gravemente Enfermo", "Prática Supervisionada de Enfermagem na Saúde da Criança", "Prática Supervisionada de Enfermagem em Serviço de Centro Cirúrgico", "Enfermagem em Saúde Mental e Psiquiatria", "Enfermagem na Saúde da Criança I e II", "Saúde Coletiva" e "Enfermagem na Saúde da Mulher I e II". Os critérios de exclusão foram: a) não ser aluno regular do curso de graduação em enfermagem; b) estar em atividade domiciliar ou em licença saúde; c) ter realizado prática supervisionada, tendo como professora de prática a pesquisadora assistente.

O contexto de coleta de dados foi no laboratório de informática de uma instituição de ensino superior, em que é equipada com um computador por aluno, no qual o simulador poderia ser acessado individualmente.

Os estudantes realizaram a resolução do estudo de caso a partir do simulador virtual, os cálculos de cada um dos elementos que o estruturam foram realizados pelo simulador virtual e a pesquisadora obteve o escore total de cada categoria. Na construção do simulador virtual, foram elaborados valores referentes a cada um dos elementos constituintes que, ao serem somados, resultam no escore da categoria. Ao passo que, ao finalizar a simulação virtual, o aluno respondia o instrumento sobre como foi a sua perspectiva durante o uso da ferramenta, ou seja, como ele acredita ter realizado o percurso cognitivo durante a resolução do caso clínico.

O escore de cada categoria foi construído a partir da média do quantitativo de itens que a compunha, pois cada categoria possui o mesmo "peso" para análise estatística, sendo este estruturado em dois, três ou mais elementos.

Para realizar a análise do percurso cognitivo do estudante durante a aplicação do simulador virtual, foram utilizadas as categorias que compõem o pensamento crítico, sendo consideradas para a identificação do raciocínio clínico. Neste estudo, entende-se 
por percurso cognitivo, a trajetória percorrida no simulador para a resolução do caso clínico, com as respostas nas etapas de identificação dos sinais e sintomas, dos diagnósticos prioritários e posteriormente, na elaboração do plano de cuidados com as intervenções de enfermagem.

A análise das variáveis quantitativas foram descritas por média e desvio-padrão ou mediana e amplitude interquartílica, e as variáveis categóricas foram descritas por frequências absolutas e relativas. Para a comparação dos escores entre as respostas do simulador e as do estudante, o teste de Wilcoxon foi aplicado. Adotou-se o nível de significância de 5\%. As análises foram realizadas no programa Statistical Package for the Social Sciences (SPSS) versão 21.0.

$\mathrm{O}$ projeto foi aprovado pela instituição proponente (CAAE 18019819.7.0000.5347), e pela instituição coparticipante (CAAE: 18019819.7.3001.5348), os estudantes que aceitaram participar, assinaram o Termo de Consentimento Livre e Esclarecido.

\section{1 Descrição do simulador virtual Health Simulator}

O Health Simulator é um simulador de casos clínicos do tipo paciente virtual (Orton; Mulhausen, 2008), que tem como objetivo reproduzir cenários reais, proporcionando o conhecimento de práticas profissionais da saúde, de modo a permitir acesso a prontuários clínicos, exames físicos e complementares, para que o aluno possa definir um ou mais diagnósticos e, a partir deste(s), indicar uma ou mais condutas. A modelagem do conhecimento do simulador é representada pelo especialista em uma rede bayesiana, por meio da qual são desenvolvidos casos clínicos (Pinheiro et al., 2015).

O aluno abre o simulador e é apresentada a tela inicial, na qual é preciso colocar os seus dados de identificação, como nome e senha de acesso. Neste estudo, os estudantes foram identificados por números, e não foi necessário cadastrar a senha, e posteriormente, eram direcionados para a tela de acesso ao simulador (Figura 1).

Figura 1 - Interface de acesso ao Health Simulator.
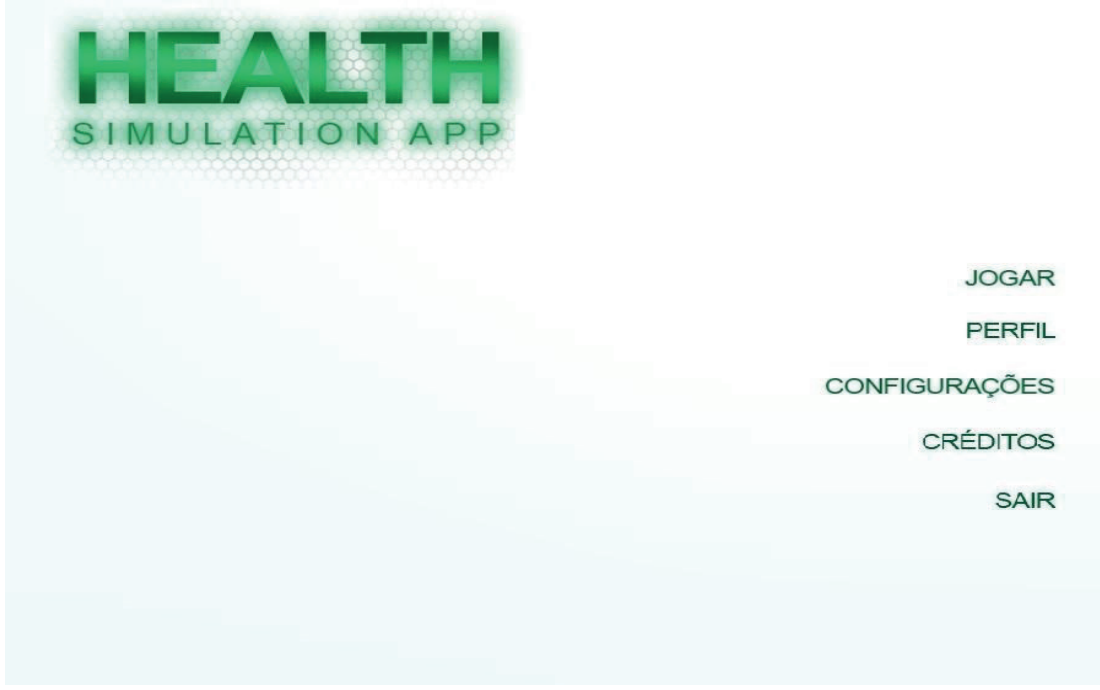

Posteriormente, um avatar randomizado pelo próprio simulador a partir das características cadastradas pelos estudantes inicia a anamnese e o exame físico do paciente em um cenário de internação clínica. A partir dessa interação, é disponibilizada 
ao aluno a ficha do paciente com o caso clínico (Figura 2), que foi cadastrada previamente pela pesquisadora na interface administrativa.

Figura 2 - Ficha do paciente com o caso clínico no Health Simulator.

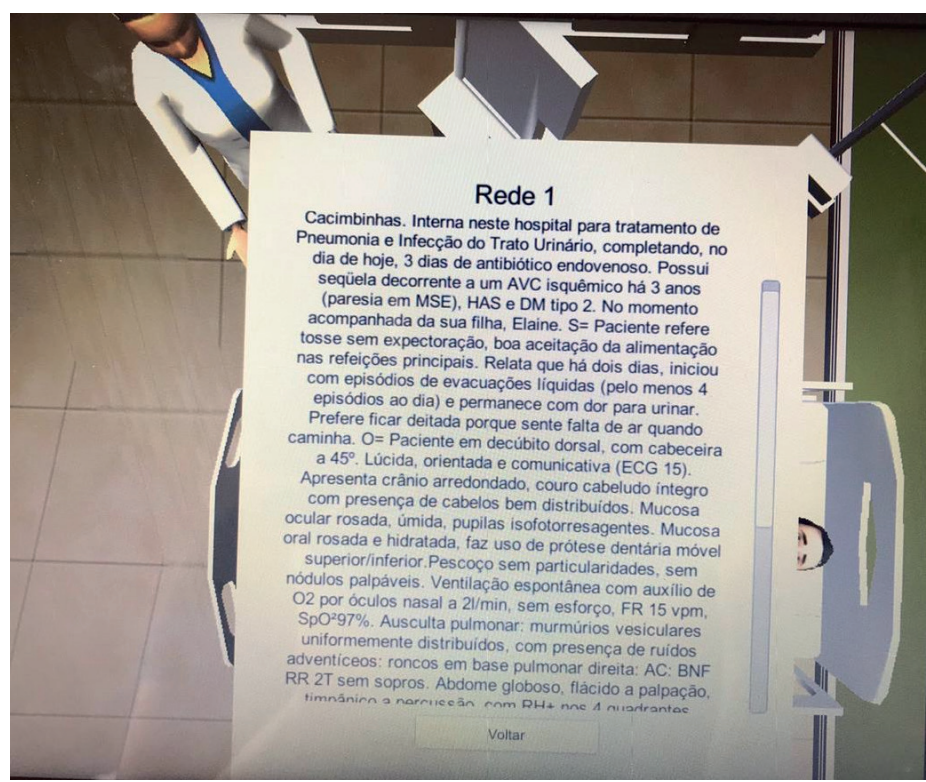

Tendo em vista os dados coletados anteriormente, o aluno deveria elencar três diagnósticos em ordem de prioridade. Após a escolha do primeiro diagnóstico, ele deveria escolher intervenções específicas para aquela condição clínica. Entretanto, antes de escolher as condutas, ele poderia realizar perguntas mais específicas a fim de aprofundar o conhecimento sobre o estado de saúde e confirmar as características definidoras e os fatores relacionados aos diagnósticos (Figura 3). Esses questionamentos eram respondidos pelo avatar paciente, cujas respostas haviam sido previamente cadastradas na interface administrativa.

Figura 3 - Tela de perguntas no Health Simulator.

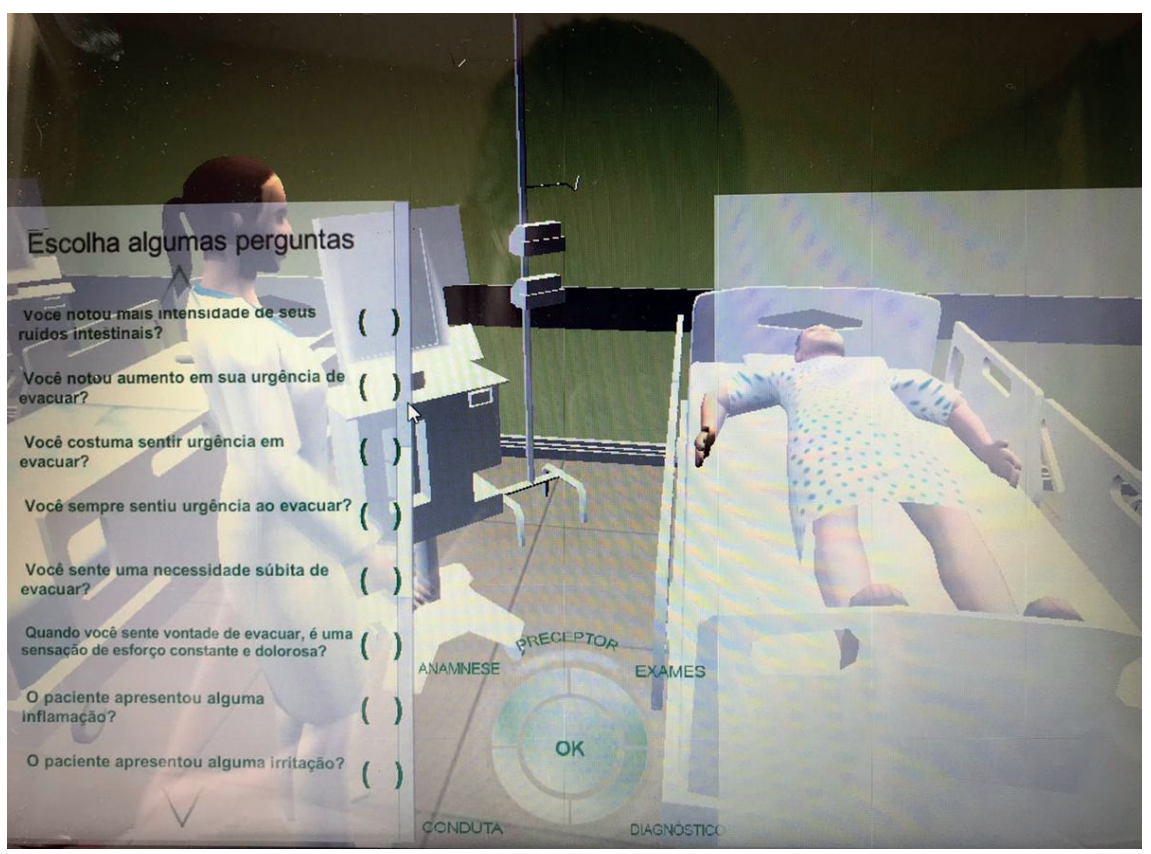


Após realizar os questionamentos considerados pertinentes, o aluno elencava as intervenções e condutas específicas para o caso e, depois de concluir o atendimento, era remetido a uma tela na qual poderia visualizar o resumo da resolução do caso, com as respostas que ele havia assinalado durante a realização do processo de enfermagem.

Cada vez que o usuário realiza uma partida do jogo, os dados referentes ao seu percurso dentro do simulador ficam registrados em um arquivo único, com os dados de identificação preenchidos pelo aluno no momento do cadastro inicial. A partir dessas informações, a pesquisadora realizou uma das partes da coleta de dados, preenchendo um instrumento específico sobre os temas/categorias pré-definidos das habilidades de pensamento crítico.

A fim de descrever a estrutura de algumas redes bayesianas que compuseram o simulador, foram desenvolvidos manuais de consulta. Eles foram elaborados pelos acadêmicos e professores dos cursos de enfermagem e de ciência da computação, da instituição co-participante, com base na taxonomia Diagnósticos de Enfermagem da Nanda International (Nanda, 2018) e Ligações entre Nanda NIC e NOC (Johnson, 2009).

\section{Resultados e Discussão}

Os dados da caracterização da amostra evidenciam que os 40 estudantes (100\%) que participaram do estudo têm idades entre 21,7 e 37,9 anos, são em sua maioria do sexo feminino, $19(47,5 \%)$ estão matriculados no $6^{\circ}$ semestre, $13(32,5 \%)$ no $7^{\circ}$ semestre e oito $(20 \%)$ no $8^{\circ}$ semestre. No que se refere ao fato de possuírem experiência como técnico em enfermagem, 17 (42,5\%) têm essa formação, e o tempo de atuação destes é de nove anos.

No que se refere ao fato de já terem realizado simulação virtual previamente, 39 $(97,5 \%)$ estudantes nunca haviam utilizado essa ferramenta, e um aluno $(2,5 \%)$ já havia tido acesso a ela há aproximadamente um ano, mas não com foco educativo, e sim para entretenimento. Quando questionados se acreditavam que a simulação virtual poderia contribuir para a sua formação como enfermeiro, os 40 (100\%) responderam que sim.

Para analisar o percurso cognitivo do estudante durante a aplicação da simulação realística e do simulador virtual, foram utilizadas as categorias de análise propostas por Bittencourt e Crossetti (2013). Os dados identificados por meio do simulador, foram confrontados com as respostas dos estudantes.

O raciocínio clínico é uma expressão genérica que geralmente expressa os processos pelos quais as informações são obtidas e interpretadas para chegar às conclusões necessárias para o cuidado em saúde (Menezes et al., 2015). As respostas do raciocínio clínico do estudante durante a simulação virtual, com a análise da trajetória cognitiva registrada a partir desta, foram comparadas com as respostas dos acadêmicos sobre como acreditam que foi o seu percurso cognitivo para a resolução do caso clínico, sendo analisado a partir dos elementos que estruturam o pensamento crítico.

A seguir, são enfatizadas as variáveis que foram estatisticamente significantes $(\mathrm{p} \leq 0,05)$. Isto é, considerou-se estatisticamente significantes aqueles aspectos que se diferenciaram nas duas avaliações (Tabela 1).

Ao analisarmos as respostas quantitativas do raciocínio clínico registradas no simulador e como o estudante percebeu-se na realização do caso simulado diante da primeira categoria - a Capacidade de análise -, os resultados demonstram que o raciocínio clínico dos estudantes não está voltado para a investigação clínica, pois é necessário que sinais e sintomas sejam elucidados com o paciente a fim de que diagnósticos prioritários sejam elencados. Os estudantes acreditaram que acertaram a primeira rede de diagnóstico (que identifica a prioridade de atendimento), mesmo não tendo conseguido marcar a rede que continha o diagnóstico correto $(\mathrm{p}<0,001)$. 
Tabela 1 - Comparativo das categorias que compõem Elementos do raciocínio clínico com as respostas do simulador e do estudante.

\begin{tabular}{|c|c|c|c|}
\hline \multirow{2}{*}{$\begin{array}{l}\text { Elementos do } \\
\text { raciocínio clínico }\end{array}$} & Simulador & Estudante & \multirow[t]{2}{*}{$\mathbf{p}$} \\
\hline & Mediana (P25-P75) & Mediana (P25-P75) & \\
\hline \multicolumn{4}{|l|}{ Capacidade de análise } \\
\hline Abriu a ficha do paciente & $100(100-100)$ & $100(100-100)$ & 1,000 \\
\hline Acertou a primeira rede de diagnósticos & $0(0-100)$ & $100(100-100)$ & $<0,001^{*}$ \\
\hline Acertou as intervenções & $65(25-95)$ & $80(50-80)$ & 0,069 \\
\hline Realizou perguntas específicas (corretas) & $50(25-80)$ & $80(50-80)$ & $0,036^{*}$ \\
\hline Escore total & $63,8(39,4-90)$ & $85(75-90)$ & $0,001^{*}$ \\
\hline \multicolumn{4}{|l|}{ Conhecimento técnico-científico } \\
\hline Acertou os diagnósticos & $50(0-80)$ & $80(50-80)$ & $0,017 *$ \\
\hline Hesitou ao escolher diagnósticos & $50(0-50)$ & $50(50-50)$ & 0,333 \\
\hline Escore total & $50(25-65)$ & $65(42,5-65)$ & 0,054 \\
\hline \multicolumn{4}{|l|}{ Raciocínio lógico } \\
\hline Realizou perguntas específicas (corretas) & $50(25-80)$ & $80(50-80)$ & $0,007^{*}$ \\
\hline Hesitou ao realizar as perguntas & $50(0-50)$ & $50(0-100)$ & 0,078 \\
\hline Escore total & $50(25-65)$ & $65(40-86,3)$ & $0,012 *$ \\
\hline Aplicação de padrões & $0(0-0)$ & $0(0-0)$ & 1,000 \\
\hline Discernimento & $50(25-80)$ & $80(50-80)$ & $0,004^{*}$ \\
\hline \multicolumn{4}{|l|}{ Experiência clínica } \\
\hline Semestre que está cursando & $50(50-50)$ & $50(50-50)$ & 1,000 \\
\hline Vivenciou casos clínicos semelhantes & $50(25-85)$ & $50(25-85)$ & 1,000 \\
\hline Escore total & $52,5(37,5-75)$ & $52,5(37,5-75)$ & 1,000 \\
\hline \multicolumn{4}{|l|}{ Conhecimento sobre o paciente } \\
\hline Abriu a ficha do paciente & $100(100-100)$ & $100(100-100)$ & 0,317 \\
\hline Realizou perguntas específicas (corretas) & $50(31,3-80)$ & $80(50-100)$ & $0,015^{*}$ \\
\hline Hesitou ao realizar as perguntas & $50(12,5-50)$ & $50(50-100)$ & 0,051 \\
\hline Escore total & $66,7(58,3-81,7)$ & $76,7(66,7-93,3)$ & $0,020^{*}$ \\
\hline
\end{tabular}

*Considerado estatisticamente significativo

Este resultado assemelha-se ao item "Realizar perguntas corretas", pois durante a investigação é necessário que o profissional realize questionamentos relevantes para a 
identificação dos sinais e sintomas, no entanto, os sujeitos acreditaram ter realizado a investigação clínica suficiente, embora as respostas do simulador tenham evidenciado o contrário $(\mathrm{p}=0,036)$. Destaca-se a importância de realizar perguntas específicas para a condição clínica do paciente, pois durante a investigação, é necessário que o profissional realize questionamentos relevantes para a identificação dos sinais e sintomas, características definidoras e fatores relacionados. Nesse contexto, os sujeitos acreditaram ter realizado a investigação clínica suficiente, mas as respostas do simulador evidenciam o contrário.

A segunda categoria refere-se ao "Conhecimento técnico-científico", relativamente ao qual os estudantes acreditam que acertaram todas as redes de diagnóstico, mesmo não tendo conseguido elencá-las corretamente $(\mathrm{p}=0,017)$. Mais uma vez, verificou-se que os alunos possuem lacunas nesta categoria.

As respostas nessa segunda categoria, que se refere ao "Conhecimento técnicocientífico", evidenciam nos resultados que os estudantes acreditam ter acertado todas as redes de diagnóstico, mesmo não tendo conseguido elencá-las corretamente. Desse modo, verifica-se que possuem lacunas nessa categoria, pois não conseguem compreender e relacionar o conhecimento teórico com as informações presentes na descrição da anamnese e exame físico do paciente virtual, desta forma, não identificam qual seria o diagnóstico de enfermagem prioritário para a condição clínica.

Com relação ao conhecimento técnico-científico, considera-se este importante por auxiliar no conhecimento de fisiopatologia para compreender e relacionar sinais e sintomas, favorecendo a comparação de situações clínicas com dados da literatura para avaliar as manifestações clínicas. A tomada de decisão clínica, diagnóstica ou terapêutica, consiste nos processos do pensamento, que compõem o raciocínio clínico (Carvalho; Oliveira-kumakura; Morais, 2017).

Esse resultado da categoria anterior, assemelha-se ao identificado no item "Raciocínio lógico", pois neste último os estudantes acreditaram estar realizando as perguntas específicas para o caso clínico, dado que é refutado a partir dos resultados verificados pela simulação virtual $(\mathrm{p}=0,007)$. Nesta situação, o escore do raciocínio lógico verificado pelo simulador foi menor do que o identificado pelos estudantes $(p=0,007)$.

O resultado na categoria anterior assemelha-se ao identificado na de "Raciocínio lógico", na qual os estudantes acreditaram ter realizado as perguntas específicas para o caso clínico, na busca por elucidar ou aprofundar sinais e sintomas. Entretanto, esse dado é refutado a partir dos resultados verificados pela simulação virtual, os quais revelaram que o escore de "Raciocínio lógico" verificado pelo simulador é menor do que o identificado pelos estudantes. Na categoria "Discernimento", os estudantes acreditaram ter acertado todas as redes que conduzem aos diagnósticos de enfermagem. Os dados permitiram identificar que o raciocínio clínico dos estudantes nesta categoria não foi suficiente durante a realização da simulação virtual $(\mathrm{p}=0,004)$, embora os alunos acreditassem possuir tal discernimento.

Na pesquisa de Bittencourt e Crossetti (2013), na habilidade de pensamento crítico, a categoria "Discernimento" foi uma das menos citadas, sendo lembrada por dois sujeitos. Para os participantes, o discernimento se faz presente no processo do diagnóstico em enfermagem por possibilitar a reflexão de situações clínicas com base na avaliação dos dados. Essa avaliação possibilita o julgamento de diagnósticos de enfermagem prioritários.

Em outro estudo, que buscou analisar os elementos estruturais do pensamento crítico definidos pelos enfermeiros de serviços de emergência, constatou-se que o conhecimento técnico-científico e o raciocínio clínico foram alguns dos elementos elencados como prioritários pelos participantes (Crossetti et al., 2014). 
No quesito "Conhecimento sobre o paciente", os estudantes responderam que realizaram as perguntas corretas, mas as respostas do simulador evidenciam que não $(p=0,015)$. Essa insegurança no momento de realizar os questionamentos aos pacientes também foi identificada na pesquisa de Forsberg e colaboradores (2019), na qual os estudantes relataram incertezas sobre as perguntas que deveriam fazer na entrevista do paciente e sobre quais exames deveriam avaliar para estabelecer os diagnósticos, ou relataram que se sentiram certos sobre o diagnóstico esperado, mas sem saber como respaldá-lo.

\section{Conclusões}

Os resultados deste estudo puderam evidenciar que o raciocínio clínico dos estudantes de enfermagem possui lacunas de conhecimento diante da resolução do caso clínico. Percebeu-se que acreditam ter maiores níveis de conhecimento dos elementos que compõem o pensamento crítico, e que isso é refutado pelo simulador virtual.

As contribuições deste estudo consistem no fato de que o exercício de realizar casos clínicos em um ambiente simulado, permite ao estudante exercitar os conhecimentos sem que haja risco ao paciente, pois a realização da simulação permite aos estudantes a possibilidade de errar sem causar dano. Ou seja, eles podem exercitar a tomada de decisão clínica sem o receio de ocasionar alguma deterioração clínica ou modificação negativa na condição de saúde do paciente, promovendo um cuidado mais acurado e com foco na segurança do paciente.

Emerge a necessidade de realização de mais estudos futuros sobre a utilização de simulação virtual, a fim de auxiliar na aprendizagem do pensamento crítico, pois, dessa forma, podem exercitar o raciocínio clínico, resolver diferentes problemas e aprender a sintetizar fatos e identificar situações de risco e prioridades clínicas.

\section{Referências}

BETTS L.; SCHMID J.; SIVARAMALINGAM S.; VERKUYL M. Using Virtual Interactive Digital Simulator to Enhance Simulation Experiences for Undergraduate Nursing Students. Nursing Education Perspectives, v.41, n.3, p.193-194, 2020.

Disponível em: <https://pubmed.ncbi.nlm.nih.gov/30807501/.

BITTENCOURT, G. K. G. D.; CROSSETTI, M. G. O. Habilidades de pensamento crítico no processo diagnóstico em enfermagem. Revista Escola de Enfermagem da USP, São Paulo, v. 47, n. 2, p. 341-347, 2013. Disponível em: $<$ http://www.scielo.br/scielo.php?script=sci_arttext\&pid=S0080$62342013000200010 \& \operatorname{lng}=e n \& n r m=i s o>$. Acesso em 29 jan. 2020.

BEZ, M. et al. Health Simulator: um simulador de casos de estudo para a área da saúde. Revista Observatório, Palmas, v. 4, n.3, p. 283-306, 2018.

CARBOGIM, F. C. et al. Modelo de ensino ativo para o desenvolvimento do pensamento crítico. Revista Brasileira de Enfermagem, v. 72, n 1, p. 305-10, 2019.

CARVALHO, E. C.; OLIVEIRA-KUMAKURA, A. R. S.; MORAIS, S. C. R. V. Raciocínio clínico em enfermagem: estratégias de ensino e instrumentos de avaliação. Revista Brasileira de Enfermagem, Brasília, v. 70, n. 3, p. 662-668, 2017. Disponível em: $\quad<\mathrm{http}: / / \mathrm{www}$.scielo.br/scielo.php?script=sci_arttext\&pid=S0034$71672017000300662 \& \operatorname{lng}=$ en\&nrm=iso $>$. Acesso em: 23 jan. 2020. 
CROSSETTI, M. G. O. et al . Elementos estruturais do pensamento crítico de enfermeiros atuantes em emergências. Revista Gaúcha de Enfermagem, Porto Alegre, v. 35, n. 3, p. 55-60, 2014. Disponível em: $<$ http://www.scielo.br/scielo.php?script=sci_arttext\&pid=S1983-

14472014000300055\&lng=en\&nrm=iso $>$. Acesso em: 29 jan. 2020.

DUBOVI, I. Online computer-based clinical simulations: the role of visualizations. Clinical Simulation in Nursing, n. 33, p. 35-41, 2019.

FITZPATRICK, J. J.; SMITH, M. J. Research on clinical decision making: challenges for professional disciplines. Applied Nursing Research, v. 26, n. 4, p. 161, 2013.

FORSBERG, E. et al. Virtual patient cases for active student participation in nursing education - Students' learning experiences. Creative Education, v. 10, p. 1475-1491, 2019.

GALLAGHER-LEPAK, S. Fundamentos do diagnóstico de enfermagem. In: NANDA I. Diagnósticos de enfermagem da NANDA: definições e classificação 2018-2020. Porto Alegre: Artmed, 2018.

JOHNSON, M. Ligações entre NANDA, NOC e NIC: diagnósticos, resultados e intervenções de enfermagem. In: JOHNSON, M. et al. Ligações entre NANDA, NOC e NIC: diagnósticos, resultados e intervenções de enfermagem. Porto Alegre: Artmed, 2009.

MÁRTIRES, M. et al. Promoção de competências de pensamento crítico em estudantes de enfermagem. Revista Lusófona de Educação, v. 44, n. 44, p. 159-172, 2019.

MENEZES, S. S. C. de et al. Clinical reasoning in undergraduate nursing education: a scoping review. Revista da Escola de Enfermagem da USP, São Paulo, v. 49, n. 6, p. 1032-1039, 2015. Disponível em: $<$ http://www.scielo.br/scielo.php?script=sci_arttext\&pid=S0080-

62342015000601032\&lng=en\&nrm=iso>. Acesso em: 23 jan. 2020.

NANDA, I. Diagnósticos de enfermagem da NANDA: definições e classificação 20182020. Porto Alegre: Artmed, 2018.

ORTON, E.; MULHAUSEN, P. E-learning virtual patients for geriatric education. Gerontology \& Geriatrics Education, v. 28, n. 3, p.73-88, 2008.

PINHEIRO, D. et al. Redes bayesianas como geração de conhecimento para games. GamePad, Novo Hamburgo, v. 1, p. 1-12, 2015.

SILVEIRA, M.; COGO, A. Contribuições das tecnologias educacionais digitais no ensino de habilidades de enfermagem: revisão integrativa. Revista Gaúcha de Enfermagem, v. 38, n. 2, 2017. Disponível em: <http://dx.doi.org/10.1590/1983-1447.2017.02.66204>. Acesso em: 31 mar. 2020. 